\section{AUTOMATED TRB LOCUS HAPLOTYPE ANALYSIS BY LONG-AMPLICON TCRB CHAIN SEQUENCING FOR POTENTIAL IMMUNE-RELATED ADVERSE EVENTS BIOMARKER RESEARCH}

Jennifer Burke*, Frances Chen, Jiajie Huang, Geoffrey Lowman, Timothy Looney, Marina Sedova. Thermo Fisher Scientific, South San Francisco, CA, USA

Background Identifying potential predictive biomarkers for immune related adverse events (irAEs) following checkpoint blockade inhibition (CPI) remains an outstanding goal of immune-oncology translational research. Polymorphism with the $\mathrm{T}$ cell receptor variable gene (TRBV) has been proposed as a potential risk factor for irAEs owing to a potential link between TRBV polymorphism and chronic autoimmune disease. Efforts to interrogate the potential biomarker utility of TRBV polymorphism have been hampered by the repetitive nature of the TRB locus. Our research has demonstrated a method for inferring TRB locus haplotypes from long-amplicon TCRB chain sequencing data, which we used to identify major haplotype groups in from nucleic acid. Here we present our research for a potential automated method for haplotype group assignment from TCRB chain sequencing data.

Methods Rearranged TCRB chains from 10 blood samples were amplified and sequenced from $25 \mathrm{ng}$ peripheral blood total RNA via the Oncomine ${ }^{\mathrm{TM}}$ TCRB-LR assay using the Genexus $^{\mathrm{TM}}$ Integrated Sequencer. 12 samples were run per chip with 4 samples run in each lane. TCRB clonotyping and repertoire feature analysis was performed using Genexus ${ }^{\mathrm{TM}}$ analysis software. Automated haplotype group assignment was performed by generation and comparison of TRBV allele profiles to those presented previously. ${ }^{1}$ For context, TCR evenness, convergence, and haplotype group assignment were compared to values obtained following analysis of the same samples via the GeneStudio ${ }^{\mathrm{TM}}$ S5 platform and Ion Reporter ${ }^{\mathrm{TM}}$ 5.12 software.

Results TCR Evenness and convergence values were highly correlated across replicates run on the Genexus ${ }^{\mathrm{TM}}$ Integrated Sequencer (Spearman correlation $>0.95$ and $>0.70$, respectively). Evenness at equivalent clone count and convergence at equivalent sequencing depth were not significantly different across platforms (Spearman correlation >0.88). Haplotype group assignments demonstrated $100 \%$ agreement across replicates on both platforms.

Conclusions Our research has demonstrated a potential automated and reproducible method for TRB haplotype group assignment via the Oncomine ${ }^{\mathrm{TM}}$ TCR-Beta LR Assay, GX run on the Genexus ${ }^{\mathrm{TM}}$ Integrated Sequencer. Future studies will be needed to evaluate the potential biomarker utility of TRB haplotyping for the prediction of irAEs.For research use only not for diagnostic procedures.

\section{REFERENCE}

1. Looney $T$, Duose D, Lowman G, Linch E, Hajjar J, Topacio-Hall D, Xu M, Zheng J, Alshawa A, Tapia C, Stephen B, Wang L, Meric-Bernstam F, Miller L, Glavin A, Lin L, Gong J, Conroy J, Morrison C, Hyland F, Naing A. Haplotype Analysis of the T-Cell Receptor Beta (TCRB) Locus by Long-amplicon TCRB Repertoire Sequencing. J Immunother Precis Oncol 2019;2:137-143.

http://dx.doi.org/10.1136/jitc-2020-SITC2020.0069

\section{BEYOND PD-L1: NOVEL PD-1 BIOMARKERS IDENTIFIED BY DRIVING T CELL DYSFUNCTION IN VITRO}

${ }^{1}$ Simarjot Pabla*, ${ }^{1}$ Tenzing Khendu, ${ }^{1}$ Dhan Chand, ${ }^{1}$ Bulent Aksoy, ${ }^{1}$ Benjamin Duckless, ${ }^{1}$ Andrew Basinski, ${ }^{1}$ Cailin Joyce, ${ }^{2}$ Thomas Horn, 'Lukasz Swiech, ${ }^{3}$ Jeremy Waight, ${ }^{1}$ David Savitsky, 'Jennifer Buell. ${ }^{1}$ AGENUS, INC., Lexington, MA, USA; ${ }^{2}$ Gilead, Foster City, California, USA; ${ }^{3} G S K$, King of Prussia, Pennsylvania, USA

Background Anti-PD-1 therapies have achieved durable clinical responses in a wide range of malignancies, but responses are limited to a small subset of patients. Expression of PD-L1 on tumor cells by immunohistochemistry (IHC) has been applied as a companion diagnostic for anti-PD-1 therapy. However, recent studies have called in to question the reliability of this method to predict response.

Methods Here we developed a novel platform that integrates in vitro pharmacogenomic and functional data with clinical pharmacodynamic responses to immunotherapy using proprietary in silico approaches. The data originate from a long-term co-culture of primary antigen-specific $\mathrm{T}$ cells and cancer cells which drives $\mathrm{T}$ cells to a terminally dysfunctional, PD-1 refractory state. $\mathrm{T}$ cell effector functions and gene expression changes were monitored in the presence or absence of antiPD-1 antibody or genetic knockouts. RNA expression signatures were refined with a randomized sliding window approach to generate a deep learning neural network for PD1 response prediction.

Results We defined five $\mathrm{T}$ cell states associated with distinct phenotypic and molecular features - naïve, active, effector, transition and dysfunction. Among the genes that were selectively expressed in the dysfunction state, we identified a 96gene signature that is closely associated with clinical outcomes to anti-PD-1 therapy. In PD-1 treated patients across multiple solid tumor indications, this signature correlates with objective response rate and outperforms traditional metrics such as tumor mutation burden or PD-L1 IHC signal. Moreover, this signature combines with tumor sequencing data to generate a powerful machine-learning model that predicts anti-PD-1 responses in metastatic melanoma patients with significantly higher accuracy than PD-L1 IHC. Having established that the $\mathrm{T}$ cell states in our co-culture relate to clinical outcomes, we leveraged the system to investigate the molecular basis for PD-1 responses. Single cell mapping of transition state $\mathrm{T}$ cells in the presence of anti-PD-1 revealed an expanded population of $\mathrm{T}$ cells that co-expresses PD-1, TIGIT and activation markers. Likewise, PD-L1 knockout on cancer cells identified the TIGIT ligand, CD155, as a potential tumor escape mechanism to anti-PD-1 therapy. Consistent with this, the combination of PD-1 and TIGIT blockade enhanced T cell cytotoxicity of tumor cells relative to monotherapies.

Conclusions Agenus' $\mathrm{T}$ cell dysfunction platform combines deep in vitro profiling and AI-based approaches to predict clinical outcomes. Here, we defined a predictive biomarker signature that outperforms standard PD-L1 IHC. Further, we identified known (TIGIT) and potentially novel combination partners predicted to enhance the durability of anti-PD-1 responses.

Ethics Approval Not Applicable

Consent Not Applicable

http://dx.doi.org/10.1136/jitc-2020-SITC2020.0070 